\title{
Ideação suicida em mulheres e violência por parceiro íntimo
}

\author{
Suicidal ideation in women and intimate partner violence
}

Ideación suicida en mujeres y violencia de pareja

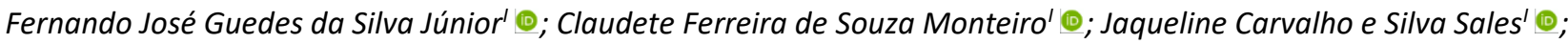

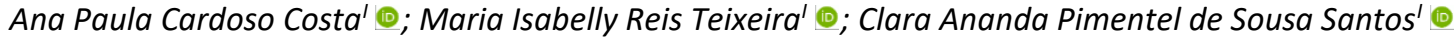

'Universidade Federal do Piauí, Teresina, PI, Brasil

\begin{abstract}
RESUMO
Objetivo: investigar a associação entre ideação suicida e violência por parceiro íntimo em mulheres. Método: estudo analítico e transversal, desenvolvido em Unidades Básicas de Saúde do Piauí-Brasil, com 369 mulheres adultas e com histórico de relacionamento. Os dados foram coletados de agosto de 2015 a março de 2016, por meio do Revised Conflict Tactics Scales e Self-Reporting Questionnaire, e analisados no software Statistical Package for the Social Science, versão 20.0. Resultados: verificou-se que $65,3 \%$ das mulheres sofreram violência por parceiro íntimo, $61,0 \%$ foi vítima de violência psicológica, $32,2 \%$ física, $18,7 \%$ moral e $17,1 \%$ sexual. Identificou-se associação entre ideação suicida e violência por parceiro íntimo $(p=0,000)$, violência psicológica $(p=0,001)$ e moral $(p=0,000)$. Ser vítima de violência por parceiro íntimo aumenta 4,35 vezes as chances de as mulheres pensarem em cometer atos contra a própria vida. Conclusão: a ideação suicida está associada a violência por parceiro íntimo, violência psicológica e moral.

Descritores: Saúde da Mulher; Saúde Mental; Violência por Parceiro Íntimo; Ideação Suicida.
\end{abstract}

\section{ABSTRACT}

Objective: to investigate the association between suicidal ideation and intimate partner violence in women. Method: analytical and cross-sectional study carried out in Basic Health Units in Piauí, Brazil, with 369 adult women and with a relationship history. Data were collected from August 2015 to March 2016 using the Revised Conflict Tactics Scales and Self-Reporting Questionnaire, and analyzed using the Statistical Package for the Social Science software, version 20.0. Results: $65.3 \%$ of the women were found to have suffered intimate partner violence; $61.0 \%$, psychological violence, $32.2 \%$, physical violence, $18.7 \%$, moral violence, and $17.1 \%$, sexual violence. An association was identified between suicidal ideation and intimate partner violence $(p$ $=0.000)$, psychological violence $(p=0.001)$, and moral violence $(p=0.000)$. Victims of intimate partner violence were 4.35 times more likely to think of committing acts against their own lives. Conclusion: suicidal ideation is associated with intimate partner violence, psychological and moral violence.

Descriptors: Women's health; Mental Health; Intimate Partner Violence; Suicidal Ideation.

\section{RESUMEN}

Objetivo: investigar la asociación entre ideación suicida y violencia de pareja en mujeres. Método: estudio analítico y transversal, realizado en Unidades Básicas de Salud de Piauí-Brasil, con 369 mujeres adultas y con antecedentes de parentesco. Los datos se recopilaron desde agosto de 2015 hasta marzo de 2016, utilizando el Revised Conflict Tactics Scales y Self-Reporting Questionnaire, y se analizaron utilizando el software Statistical Package for the Social Science, versión 20.0. Resultados: se encontró que el 65,3\% de las mujeres sufrió violencia de pareja íntima, el 61,0\% fue víctima de violencia psicológica, 32,2\% física, $18,7 \%$ moral y $17,1 \%$ sexual. Se identificó asociación entre ideación suicida y violencia de pareja íntima $(p=0,000)$, violencia psicológica $(p=0,001)$ y violencia moral $(p=0,000)$. Ser víctima de violencia de pareja aumenta 4,35 veces las posibilidades de que las mujeres piensen en cometer actos contra su propia vida. Conclusión: la ideación suicida está asociada con la violencia de pareja, violencia psicológica y moral.

Descriptores: Salud de la Mujer; Salud mental; Violencia de Pareja; Ideación Suicida.

\section{INTRODUÇÃO}

A problemática do comportamento suicida tem sido discutida em diversos contextos e sob várias faces ${ }^{1-3}$. Esse fenômeno está relacionado à conduta de autoagressão e desejo de morte. A ideação suicida integra esse comportamento, juntamente com o planejamento, a tentativa e a morte por suicídio ${ }^{4}$.

No universo feminino, a ideação suicida pode estar relacionada à violência, sobretudo, àquela praticada pelo companheiro ${ }^{5}$. Nesse sentido, a violência por parceiro íntimo (VPI) é definida como quaisquer danos associados à relação conjugal, sejam eles físicos, psicológicos e sexuais, dentre outros ${ }^{6,7}$.

Globalmente, a VPI tem afetado milhões de mulheres, causado problemas de saúde, incapacidades e morte ${ }^{8}$. Do total de homicídios femininos, $38 \%$ foram atribuídos à $\mathrm{VPI}^{9}$. Além disso, reflete múltiplas adversidades na saúde física e mental delas, sua ocorrência corrobora para o desenvolvimento de transtornos mentais, a exemplo: depressão, transtorno de estresse pós-traumático e uso abusivo de álcool e outras drogas ${ }^{10}$. 
Estudo de revisão sistemática revelou que, dentre fatores associados à VPI, estão: baixo nível de escolaridade ou socioeconômico, consumo de substâncias psicoativas, exposição anterior a outras formas de abuso ou violência, atitude de tolerância à violência, controle de comportamento pelo parceiro, abuso de substâncias e envolvimento em brigas, e falta de apoio social ${ }^{11}$.

Destaca-se que mulheres que vivenciam VPI estão mais suscetíveis a se tornarem tristes e com baixa autoestima, aspectos que prejudicam a qualidade de vida, e consequentemente, as tornam mais propensas a ter ideias de atentar contra a própria vida ${ }^{12}$. Panorama geral aponta que a população feminina já apresenta taxas mais elevadas de tentativa de suicídio quando comparada aos homens ${ }^{13}$. Não obstante, a exposição a situações de violência oferece risco aumentado para esse agravo ${ }^{14}$.

Frente ao exposto, considera-se emergente a ampliação das produções científicas brasileiras acerca do comportamento suicida com vistas a contribuir para o efetivo enfrentamento desta problemática. Dessa forma, o objetivo deste estudo é investigar associação entre ideação suicida e violência por parceiro íntimo em mulheres.

\section{MÉTODO}

Trata-se de estudo analítico e transversal, de abordagem quantitativa, desenvolvido em 72 Unidades Básicas de Saúde (UBS) de cinco municípios do Estado do Piauí: Teresina, Parnaíba, Picos, Floriano e Bom Jesus, considerados referência para as cinco macrorregiões de saúde do estado.

A população de referência foi de 347.414 mulheres, com idades entre 20 a 59 anos, nos referidos municípios ${ }^{15}$. Para o cálculo amostral considerou-se nível de confiança de $95 \%$, erro máximo de $5 \%$ e prevalência presumida de VPI de $39 \%{ }^{16}$, que determinou amostra de 369 mulheres. Realizou-se distribuição proporcional dessas mulheres nos cinco municípios de coleta, resultando em 232 investigadas na cidade de Teresina, 46 em Picos, 38 em Floriano, 36 em Parnaíba, e 17 em Bom Jesus.

Para manter o processo de aleatorização, no recrutamento das participantes, realizou-se sorteio das mulheres a serem incluídas no estudo, tendo em vista o cadastro daquelas atendidas na UBS no ano de 2014.

Foram estabelecidos como critério de inclusão: mulheres, com idade entre 20 a 59 anos, com histórico de relacionamento (namoro, casamento ou afins) e atendidas nas consultas de enfermagem nas UBSs. Foram excluídas aquelas que possuíam deficiência auditiva ou doença mental, identificada a partir de registro no prontuário. Quando uma mulher era excluída, uma nova participante era sorteada.

A coleta de dados foi realizada no período de agosto de 2015 a março de 2016 . As situações de violência foram avaliadas a partir da aplicação da Revised Conflict Tactics Scales (CTS2) ${ }^{17}$. A CTS2 foi concebida, especificamente, para identificação da violência entre indivíduos que tenham uma relação de namoro, casamento ou afins. Foram consideradas vítimas as mulheres que referiram ter sofrido pelo menos um dos 12 atos de violência apontadas pela escala ${ }^{18}$.

A ideação suicida, por sua vez, foi avaliada a partir da aplicação do Self-Reporting Questionnaire (SRQ-20), instrumento de rastreamento de transtornos mentais não-psicóticos ${ }^{19}$. Para mensurar essa variável trabalhou-se com questão específica do domínio "sintomas depressivos", que indaga se a participante "tem tido ideias de acabar com a vida?".

Foi realizado um teste piloto com $10 \%$ da amostra, com objetivo de testar os instrumentos e a habilidade dos pesquisadores. As informações adquiridas desta etapa não fizeram parte do banco de dados.

Os dados foram analisados no software Statistical Package for the Social Science (SPSS), versão 20.0. Realizou-se análises descritivas e inferenciais. Para verificar associação entre variáveis qualitativas utilizou-se o teste qui-quadrado. Quando da frequência das caselas foi menor que $20 \%$ ou menor que 5 realizou-se teste exato de Fischer. A força das associações entre as variáveis foi aferida pelo odds-ratio (OR) e intervalos de confiança (IC 95\%).

Todos os aspectos éticos foram preservados durante a realização deste estudo. A pesquisa foi aprovada pelo Comitê de Ética em Pesquisa da instituição proponente. As participantes foram esclarecidas quanto à pesquisa e assinaram o Termo de Consentimento Livre e Esclarecido.

\section{RESULTADOS}

A maioria das mulheres possuía, em média, 33,1 anos (Desvio padrão=9,9), eram pardas $(59,4 \%)$, com filhos $(70,7 \%)$ e heterossexuais (98,6\%). Tinham, em média, 10 anos de estudo (Desvio padrão=3,5), 57,3\% possuíam emprego formal, $60,4 \%$ referiram fonte de renda proveniente do próprio salário e $24,6 \%$ do bolsa família. A renda individual média foi de 799,8 reais (Desvio padrão=637,0). A Tabela 1 apresenta dados relacionados à ocorrência de violência. 
TABELA 1: Ocorrência de violência por parceiro íntimo em mulheres atendidas na Estratégia Saúde da Família. Piauí, Brasil, 2018.

\begin{tabular}{lccc}
\hline \multirow{2}{*}{ Variáveis } & \multicolumn{2}{c}{ Ocorrência de violência } & \\
\cline { 2 - 3 } & $\begin{array}{c}\text { Sim } \\
\mathbf{n ( \% )}\end{array}$ & $\begin{array}{c}\text { Não } \\
\text { n(\%) }\end{array}$ & $\begin{array}{c}\text { Total } \\
\mathbf{n}(\%)\end{array}$ \\
\hline Violência & $240(65,3)$ & $128(34,7)$ & $369(100)$ \\
Violência Psicológica & $225(61,0)$ & $144(39)$ & $369(100)$ \\
Violência Física & $119(32,2)$ & $250(67,8)$ & $369(100)$ \\
Violência Sexual & $63(17,1)$ & $306(82,9)$ & $369(100)$ \\
Violência Moral & $69(18,7)$ & $300(81,3)$ & $369(100)$ \\
\hline
\end{tabular}

Verificou-se que 65,3\% das mulheres sofreu algum tipo de violência, 61,0\% foi vítima de violência psicológica, $32,2 \%$ violência física, 18,7\% violência moral e 17,1\% violência sexual. A Tabela 2 apresenta dados sobre a associação entre ideação suicida e violência.

TABELA 2: Associação entre ideação suicida e violências por parceiro íntimo em mulheres atendidas na Estratégia Saúde da Família. Piauí, Brasil, 2018.

\begin{tabular}{|c|c|c|c|c|c|}
\hline \multirow[b]{2}{*}{ Variáveis } & \multicolumn{2}{|c|}{ Ideação suicida } & \multirow[b]{2}{*}{ p-valor } & \multirow[b]{2}{*}{ OR $\#$} & \multirow[b]{2}{*}{ IC $(95 \%)^{\# \#}$} \\
\hline & $\begin{array}{l}\text { Não } \\
\text { n(\%) }\end{array}$ & $\begin{array}{l}\text { Sim } \\
n(\%)\end{array}$ & & & \\
\hline Violências & & & $0,000 * *$ & 4,355 & $1,765-10,748$ \\
\hline Sim & $41(89,1)$ & $200(61,9)$ & & & \\
\hline Não & $5(10,9)$ & $123(38,1)$ & & & \\
\hline Violência psicológica & & & $0,001 * *$ & 0,289 & $0,131-0,640$ \\
\hline Sim & $187(57,9)$ & $38(82,6)$ & & & \\
\hline Não & $136(42,1)$ & $8(17,4)$ & & & \\
\hline Violência física & & & $0,221^{*}$ & 0,110 & $0,054-0,227$ \\
\hline Sim & $84(26)$ & $35(76,1)$ & & & \\
\hline Não & $239(74)$ & $11(23,9)$ & & & \\
\hline Violência sexual & & & $0,37^{* *}$ & 0,465 & $0,229-0,945$ \\
\hline Sim & $50(15,5)$ & $13(28,3)$ & & & \\
\hline Não & $273(84,5)$ & $33(71,7)$ & & & \\
\hline Violência moral & & & $0,000 * *$ & 0,093 & $0,048-0,184$ \\
\hline Sim & $41(12,7)$ & $28(60,9)$ & & & \\
\hline Não & $282(87,3)$ & $18(39,1)$ & & & \\
\hline Total & $323(100)$ & $46(100)$ & & & \\
\hline
\end{tabular}

Observa-se associação estatisticamente significativa entre ideação suicida e violências $(p=0,000)$. Observou-se que ser vítima de violências aumenta 4,35 vezes as chances de as mulheres pensarem em cometer atos contra a própria vida. Quanto aos tipos de violências, verificou-se que violência psicológica e moral apresentaram associação negativa com ideação suicida (OR=0,289 e 0,093, respectivamente).

\section{DISCUSSÃO}

Os resultados deste estudo se somam ao cenário estatístico que abrange a VPI e suas implicações para mulheres e para sociedade, especificamente, a relação desse fenômeno com a ideação suicida, objetos de estudo com elevada magnitude, que são alvos de preocupação mundial.

Os dados levantados por este estudo demonstram que a ocorrência de VPI, no Piauí, é quase o dobro das estimativas mundiais, pois, de acordo com a Organização Mundial da Saúde ${ }^{9}, 35 \%$ das mulheres no mundo já sofreram violência perpetrada por parceiro íntimo. Quando comparado à realidade brasileira, estudo de base populacional, também identificou ocorrência de violência inferior $(43 \%)^{20}$. 
Dado elevado de VPI, no Estado do Piauí, ratifica as teorias que explicam o fenômeno da violência sob ação do patriarcado. As desigualdades de gênero construídas socioculturalmente impactam nas relações de poder. Na realidade contextual, deste estudo, em que parcela significativa das mulheres não exerce funções remuneradas e que as desigualdades educacionais são consideráveis, a dominação é mais recorrente e a vulnerabilidade para VPI potencial.

O estabelecimento de relações de poder ainda se sobressaem e é questão central do fenômeno da violência. A opressão é uma forma de exercer o poder patriarcal dominante e, ao mesmo tempo, perpetuar as iniquidades de poder expressas nas relações desiguais de gênero ${ }^{21,22}$.

Dentre as diversas formas de VPI, a mais relatada pela amostra deste estudo foi a violência psicológica. Essa forma de expressão da violência, juntamente com a violência moral, são complexas, silenciosas e se expressam por meio de insultos ou constrangimentos à parceira, fazendo-a se sentir mal a respeito de si mesma, além de depreciações, ridicularizações ou humilhações diante de outras pessoas ${ }^{23}$. Estudo evidencia que estes tipos de violência geram danos devastadores para as vítimas, a citar: dores crônicas, síndrome do pânico, depressão, distúrbios alimentares e comportamento suicida ${ }^{24}$.

Neste estudo, verificou-se a existência de associação entre VPI, violência psicológica, moral e ideação suicida. A presença, das diversas formas de expressão da VPI aumentam a vulnerabilidade das mulheres ao comportamento suicida, que é caracterizado como problema mundial, complexo e multidimensional.

Destaca-se que, as mulheres, ao terem suas trajetórias vinculadas a questões complexas, não apenas impostas pelo relacionamento abusivo, mas, também, pela precarização de suas condições de vida e saúde pensem que a melhor solução seja sua autodestruição. Estudo aponta que a ideação e as tentativas de suicídio entre mulheres são expressivamente maiores se comparadas aos homens, uma vez que culturalmente tal ato é reprovado pela moral, mas, na maioria das vezes, entendido como única solução encontrada por mulheres para seus problemas ${ }^{25}$.

Pesquisa desenvolvida com mulheres residentes em Massachusetts verificou que as vivências de VPI física ou sexual foram significativamente associadas à ideação suicida ao longo da vida ${ }^{5}$. Estudo congênere, realizado em Nicarágua, demonstrou que mulheres que tentaram suicídio experimentaram consideravelmente mais eventos de VPI ${ }^{26}$. No Uruguai, pesquisa também constatou os efeitos negativos da agressão por parceiro íntimo à saúde mental da mulher e concluiu que a VPI se comporta como fator de risco para ideação suicida ${ }^{27}$.

Os dados encontrados nesta pesquisa são preocupantes, pois, embora se observe que as mulheres que sofrem VPI têm sido alvo de inúmeras medidas protetivas, conquistadas por meio da militância de diversos segmentos da sociedade, ainda assim permanecem em risco para atos violentos, que, por sua vez, podem repercutir em consequências graves.

Há necessidade de reflexão e ressignificação das práticas para enfrentamento de situações de violência contra a mulher ${ }^{28}$, especialmente na Atenção Primária à Saúde, tendo em vista sua atuação como porta de entrada ${ }^{29}$. Para além da identificação e tratamento, o exercício do cuidado pautado no acolhimento, escuta e estabelecimento de diálogo, são potenciais ferramentas para utilização nesse contexto ${ }^{30}$.

A violência praticada contra a mulher é, ainda, uma realidade complexa, mas fatores como a capacitação de profissionais, fortalecimento de políticas públicas e a articulação das redes de atenção à saúde, são estratégias que podem minimizar as limitações existentes, em busca de findar essa problemática que assola a população feminina ${ }^{29}$.

Merece destaque o atual contexto de pandemia de COVID-19, no qual a ocorrência de VPI tem suscitado mais discussões ${ }^{31-33}$. Neste período, a possibilidade de aumento dos episódios dessa violência se dá, em virtude de aspectos como, aumento do estresse devido a diminuição da mobilidade; ampliação das interações familiares e atividades domésticas, que repercutem em aumento das demandas de cuidado e responsabilização; desemprego; trabalho remoto; e isolamento social ${ }^{34}$

Portanto, as evidências ordenam a intensificação de rastreio da VPI em todo o território, bem como a ampliação da rede de apoio à mulher ${ }^{35}$. Todos os esforços podem ser diferenciais e devem ser empregados em busca de sanar esse fenômeno desafiador, que gera repercussões que podem culminar, até mesmo, em ideias de autodestruição.

\section{Limitações do estudo}

Apesar dos resultados relevantes do presente estudo, o desenho metodológico está implicado à limitação, pois não permite estabelecer relação de causa e efeito. Merece também ser pontuada a forma de obtenção das informações, pois, por envolver fenômeno permeado por estigma e tabu é possível que os dados estejam subestimados.

\section{CONCLUSÃO}

Observou-se que elevada proporção da amostra foi vítima de VPI, sendo mais frequente a violência psicológica, seguida da física, moral e sexual. Sofrer VPI, violência psicológica e moral demonstraram associação com ideação suicida. 
Os achados deste estudo reforçam, sobremaneira, quão devastadora pode ser a vivência de VPI no universo feminino. Assim, faz-se importante o rastreio dessas mulheres e o desenvolvimento de intervenções que focalizem nas consequências dessa violência. Atenção especial deve ser dada à prevenção da ideação suicida, visto que pode resultar em desfechos fatais.

Nesta perspectiva, destaca-se o protagonismo da atenção básica e o papel das UBS nessa articulação com o território, que pode contribuir para desenvolvimento de estratégias de enfrentamento da VPI, considerando o contexto que essas mulheres estão inseridas, seus condicionantes e determinantes sociais. A associação da ideação suicida com as violências sofridas pelas mulheres na intimidade com seus companheiros merece pesquisa e estratégias de apoio e cuidado, evitando o sofrimento e o número de mortes de mulheres.

\section{REFERÊNCIAS}

1. Pengpid S, Peltzer K. Prevalence and correlates of suicidal behaviour among a national population-based sample of adults in Zambia. S Afr J Psychiatr [Internet]. 2021 [cited 2021 Apr 15]; 27:a1566. DOI: https://doi.org/10.4102/sajpsychiatry.v27i0.1566

2. Olibamoyo O, Ola B, Coker O, Adewuya A, Onabola A. Trends and patterns of suicidal behaviour in Nigeria: Mixed-methods analysis of media reports from 2016 to 2019. S Afr J Psychiatr [Internet]. 2021 [cited 2021 Apr 15]; 27:a1572. DOI: https://doi.org/10.4102/sajpsychiatry.v27i0.1572

3. Dumith SC, Demenech LM, Carpena MX, Nomiyama S, Neiva-Silva L, Mola CL. Suicidal thought in southern Brazil: Who are the most susceptible? J Affect Disord [Internet]. 2020 [cited 2021 Apr 16]; 260:610-6. DOI: https://doi.org/10.1016/j.jad.2019.09.046

4. World Health Organization (WHO). Preventing suicide: a global imperative. Genebra: WHO; 2014.

5. Maru M, Saraiya T, Lee CS, Meghani O, Hien D, Hahm HC. The Relationship Between Intimate Partner Violence and Suicidal Ideation among Young Chinese, Korean, and Vietnamese American Women. Women Ther [Internet]. 2018 [cited 2021 Apr 15]; 41(3-4):339-55. DOI: https://doi.org/10.1080/02703149.2018.1430381

6. Meekers D, Pallin SC, Hutchinson P. Intimate partner violence and mental health in Bolivia. BMC Womens Health [Internet]. 2013 [cited 2019 Jul 15]; 13(1):1-7. DOI: https://doi.org/10.1186/1472-6874-13-28

7. Lindner SR, Coelho EBS, Bolsoni CC, Rojas PF, Boing AF. Prevalence of intimate partner physical violence in men and women from Florianópolis, Santa Catarina State, Brazil: a population-based study. Cad Saude Publica [Internet]. 2015 [cited 2019 Jul 15]; 31(4):815-26. DOI: https://doi.org/10.1590/0102-311X00159913

8. Bergmann JN, Stockman JK. How does intimate partner violence affect condom and oral contraceptive Use in the United States?: A systematic review of the literature. Contraception [Internet]. 2015 [cited 2019 Jun 03]; 91(6):438-55. DOI: https://doi.org/10.1016/j.contraception.2015.02.009

9. World Health Organization (WHO). Global and regional estimates of violence against women: prevalence and health effects of intimate partner violence and non-partner sexual violence. Genebra: WHO; 2013.

10. Stockman JK, Hayashi H, Campbell JC. Intimate partner violence and its health impact on disproportionately affected populations, including minorities and impoverished groups. J Womens Health (Larchmt) [Internet]. 2015 [cited 2019 Aug 06]; 24(1):62-79. DOI: https://doi.org/10.1089/jwh.2014.4879

11. Shahar HK, Jafri F, Zulkefli NAM, Ahmad N. Prevalence of intimate partner violence in Malaysia and its associated factors: a systematic review. BMC Public Health [Internet]. 2020 [cited 2021 Apr 16]; 20(1):1550. DOI: https://doi.org/10.1186/s12889020-09587-4

12. Santos AG, Monteiro CFS, Feitosa CDA, Veloso C, Nogueira LT, Andrade EMLR. Types of non-psychotic mental disorders in adult women who suffered intimate partner violence: an integrative review. Rev Esc Enferm USP [Internet]. 2018 [cited 2019 Jul 17]; 52:e03328. DOI: https://doi.org/10.1590/s1980-220x2017030203328

13. Ribeiro NM, Castro SS, Scatena LM, Haas VJ. Time-trend analysis of suicide and of health information systems in relation to suicide attempts. Texto Contexto Enferm [Internet]. 2018 [cited 2021 Apr 18]; 27(2):e2110016. DOI: http://dx.doi.org/10.1590/0104-070720180002110016

14. Kavak F, Aktürk Ü, Özdemir A, Gültekin A. The relationship between domestic violence against women and suicide risk. Arch Psychiatr Nurs [Internet]. 2018 [cited 2021 Apr 14]; 32(4):574-9. DOI: https://doi.org/10.1016/j.apnu.2018.03.016

15. Instituto Brasileiro de Geografia e Estatística (IBGE). Censo 2010. Rio de Janeiro (RJ): IBGE; 2013.

16. Albuquerque JBC, César ESR, Silva VCL, Espínola LL, Azevedo EB, Ferreira Filha MO. Domestic violence: sociodemographic characteristics of women registered at a family health unit. Rev Eletr Enf [Internet]. 2013 [cited 2018 Feb 11]; 15(2):382-90. DOI: http://dx.doi.org/10.5216/ree.v15i2.18941

17. Straus MA, Hamby SL, Boney-McCoy S, Sugarman DB. The revised Conflict Tactics Scales (CTS2): Development and preliminary psychometric data. Journal of Family Issues [Internet]. 1996 [cited 2018 Feb 10]; 17(3):283-316. DOI: https://doi.org/10.1177/019251396017003001

18. Moraes CL, Hasselmann MH, Reichenheim ME. Portuguese-language cross-cultural adaptation of the Revised Conflict Tactics Scales (CTS2), an instrument used to identify violence in couples. Cad Saude Publica [Internet], 2002 [cited 2018 Feb 07]; 18(1):163-76. DOI: https://doi.org/10.1590/S0102-311X2002000100017

19. Mari JJ, Williams P. A validity study of a psychiatric screening questionnaire (SRQ-20) in primary care in the city of São $\mathrm{Paulo}$. $\mathrm{Br}$ J Psychiatry [Internet]. 1986 [cited 2018 Feb 10]; 148:23-6. DOI: https://doi.org/10.1192/bjp.148.1.23 
20. Venturi G, Recamán M, Oliveira S. A mulher brasileira nos espaços público e privado. 1 ed. São Paulo: Fundação Perseu Abramo; 2004.

21. Leite FMC, Luis MA, Amorim MHC, Maciel ELN, Gigante DP. Violência contra a mulher e sua associação com o perfil do parceiro íntimo: estudo com usuárias da atenção primária. Rev Bras Epidemiol [Internet]. 2019 [cited 2019 Nov 12]; 22:e190056. DOI: https://doi.org/10.1590/1980-549720190056

22. Lucena KDT, Deininger LSC, Coelho HFC, Monteiro ACC, Vianna RPT, Nascimento JA. Analysis of the cycle of domestic violence against women. J Hum Growth Dev [Internet]. 2016 [cited 2019 Dec 03]; 26(2):139-46. DOI: http://dx.doi.org/10.7322/jhgd.119238

23. Pires MRM, Locatelli TZ, Rojas PFB, Lindner SR, Bolsoni CC, Coelho EBS. Prevalence and associated factors of psychological violence against pregnant women in capital in southern Brazil. Sau \& Transf Soc [Internet]. 2017 [cited 209 Dec 05]; 8(1):29-39. Available from: http://incubadora.periodicos.ufsc.br/index.php/saudeetransformacao/article/view/3797/4961

24. Siqueira VB, Leal IS, Fernandes FECV, Melo RA, Campos MEAL. Violência psicológica contra mulheres usuárias da atenção primária à saúde. Rev APS [Internet], 2018 [cited 2019 Dec 08]; 21(3):437-49. DOI: https://doi.org/10.34019/18098363.2018.v21.16379

25. Marquetti FR, Marquetti FC. Suicide and Feminities. Cad Pagu [Internet]. 2017 [cited 2019 Dec 16]; 49:e174921. DOI: https://doi.org/10.1590/18094449201700490021

26. Guillen Al, Panadero S, Rivas E, Vázquez JJ. Suicide attempts and stressful life events among female victims of intimate partner violence living in poverty in Nicaragua. Scand J Psychol [Internet]. 2015 [cited 2019 Oct 23]; 56 (3):349-56. DOI: https://doi.org/10.1111/sjop.12207

27. Martínez SL, Wasser AC. Depression and suicide ideation in women victims of intimate partner violence. Psicol Conoc Soc [Internet]. 2019 [cited 2020 Jan 07]; 9(1):178-204. DOI: http://dx.doi.org/10.26864/pcs.v9.n1.1

28. Morais KC, Silva KA, Gomes SC, Oliveira CAN, Silva JPX, Leite JCS. Domestic abuse: reflecting on concepts and giving new meanings to practices on primary health care. Saúde Coletiva [Internet]. 2020 [cited 2021 Apr 17]; 10(58):3903-12. DOI: https://doi.org/10.36489/saudecoletiva.2020v10i58p3903-3912

29. Pereira ACC, Cruz MAC, Rios AR, Silva BL, Andrade BG, Lima BLG, et al. Abordagem da violência doméstica contra a mulher na atenção primária à saúde: aspectos relacionados à inexperiência médica. Revista Eletrônica Acervo Saúde [Internet]. 2020 [cited 2021 Apr 18]; 12(10):e4580. DOI: https://doi.org/10.25248/reas.e4580.2020

30. Borburema TLR, Pacheco AP, Nunes AA, Moré CLOO, Krenkel S. Violence against women in the context of social vulnerability in primary health care: logging of violence in medical records. Rev Bras Med Fam Comunidade [Internet]. 2017 [cited 2021 Apr 18]; 12(39):1-13. DOI: http://dx.doi.org/10.5712/rbmfc12(39)1460

31. Lyons M, Brewer G. Experiences of Intimate Partner Violence during Lockdown and the COVID-19 Pandemic. J Fam Violence [Internet]. 2021 [cited 2021 Apr 18]; 1-9. DOI: https://doi.org/10.1007/s10896-021-00260-x

32. Agüero JM. COVID-19 and the rise of intimate partner violence. World Dev [Internet]. 2021 [cited 2021 Apr 18]; $137: 105217$. DOI: https://doi.org/10.1016/j.worlddev.2020.105217

33. Moreira DN, Costa MP. The impact of the Covid-19 pandemic in the precipitation of intimate partner violence. Int J Law Psychiatry [Internet]. 2020 [cited 2021 Apr 18]; 71:101606. DOI: https://doi.org/10.1016/j.ijlp.2020.101606

34. Oliveira W, Magrin J, Andrade A, Micheli D, Carlos D, Fernández J, Silva M, Santo M. The impact of the Covid-19 pandemic in the precipitation of intimate partner violence. Psicologia, Saúde \& Doenças [Internet]. 2020 [cited 2021 Apr 16]; 21(3):606-23. DOI: http://dx.doi.org/10.15309/20psd210306

35. Silva AF, Estrela FM, Soares CFS, Magalhães JRF, Lima NS, Morais AC, et al. Marital violence precipitating/intensifying elements during the Covid-19 pandemic. Cien Saude Colet [Internet]. 2020 [cited 2021 Apr 18]; 25(9):3475-80. DOI:

http://dx.doi.org/10.1590/1413-81232020259.16132020 